\title{
Integrated Operations of the National Ignition Facility (NIF) Optical Pulse Generation Development System
}

\author{
J. K. Crane, R. B. Wilcox, N. W. Hopps, \\ D. Browning, M. D. Martinez, B. Moran, \\ F. Penko, J. E. Rothenberg, M. Henesian, \\ C. B. Dane, and L. A. Hackel
}

This paper was prepared for submittal to the Third Annual International Conference on Solid State Lasers for Application (SSLA) to Inertial Confinement Fusion (ICF) Monterey, California June 7-12, 1998

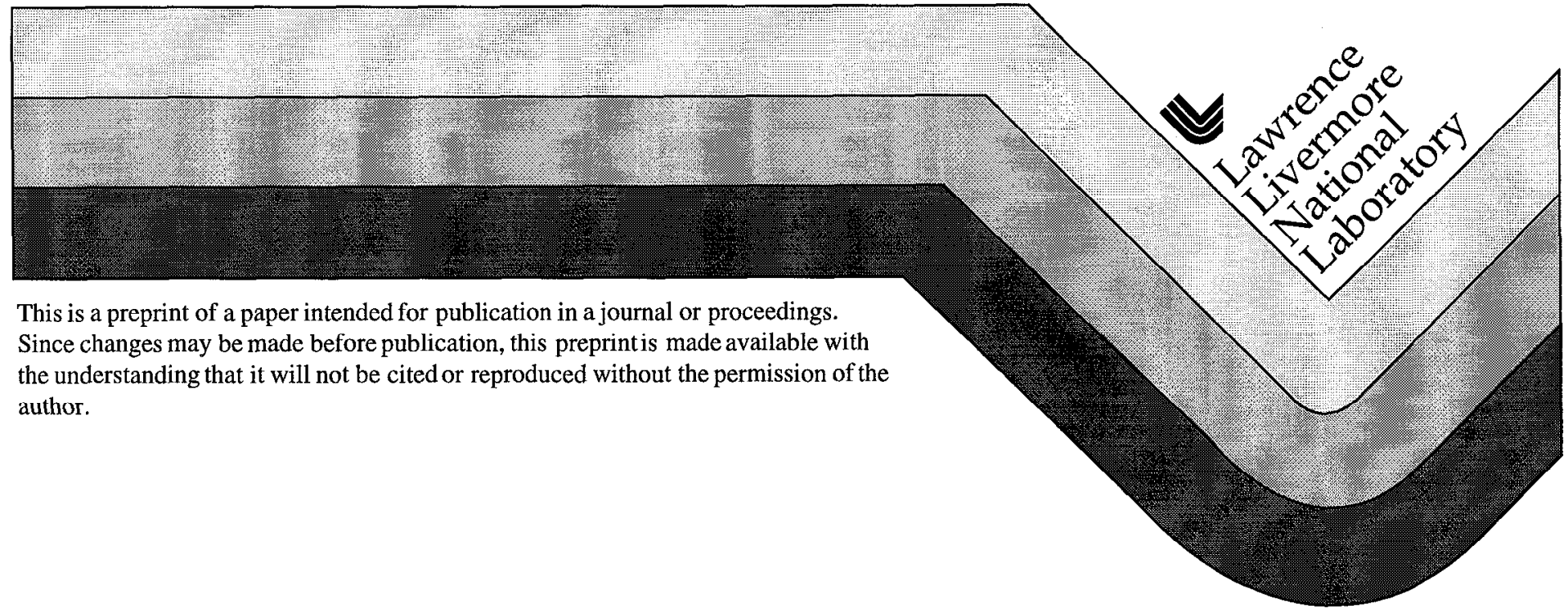


DISCLAIMER

This document was prepared as an account of work sponsored by an agency of the United States Government. Neither the United States Government nor the University of California nor any of their employees, makes any warranty, express or implied, or assumes any legal liability or responsibility for the accuracy, completeness, or usefulness of any information, apparatus, product, or process disclosed, or represents that its use would not infringe privately owned rights. Reference herein to any specific commercial product, process, or service by trade name, trademark, manufacturer, or otherwise, does not necessarily constitute or imply its endorsement, recommendation, or favoring by the United States Government or the University of California. The views and opinions of authors expressed herein do not necessarily state or reflect those of the United States Government or the University of California, and shall not be used for advertising or product endorsement purposes. 
Integrated operations of the National Ignition Facility (NIF) optical pulse generation development system

J. K. Crane, R. B. Wilcox, N. W. Hopps ${ }^{1}$, D. Browning, M. D. Martinez, B. Moran, F. Penko, J. E. Rothenberg, M. Henesian, C. B. Dane, L. A. Hackel

Lawrence Livermore National Laboratory

P. O. Box 808, Livermore, California, 94551.

${ }^{1}$ Atomic Weapons Establishment, Aldermaston, Reading, RG7 4PR.

Unitcd Kingdom.

\begin{abstract}
We describe the Optical Pulse Generation (OPG) testbed, which is the integration of the MOR and Preamplifier Development Laboratories. We use this OPG testbed to develop and demonstrate the overall capabilites of the NIF laser system front end. We will present the measured energy and power output, temporal and spatial pulse shaping capability, FM bandwidth and dispersion for beam smoothing, and measurements of the pulse-to-pulse power variation of the OPG system and compare these results with the required system performance specifications. We will discuss the models that are used to predict the system performance and how the OPG output requirements flowdown to the subordinate subsystems within the OPG system.
\end{abstract}

\title{
1. INTRODUCTION
}

The Optical Pulse Generation system (OPG) or Front-End of the NIF laser system creates and formats the pulse for the main amplifiers. The OPG is comprised of three major systems shown in the block diagram, Figure 1. The entire NIF output originates from a single pulse produced in the Master Oscillator Room (MOR). The MOR adds bandwidth for stimulated Brilluoin scattering (SBS) suppression and smoothing by spectral dispersion (SSD). The single pulse is next divided and amplified to yield 48 equal pulses to seed the rest of the NIF laser system. Each of the 48 pulses is separately shaped using an electronic arbitrary waveform generator (AWG) that drives an electrooptic, amplitude modulator.

\section{Schematic of OPG system}

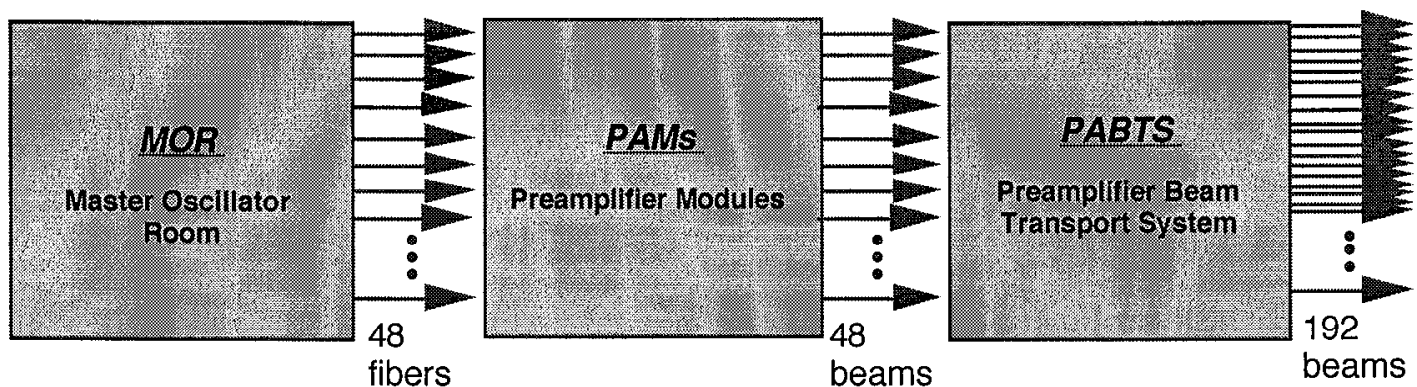

Figure 1. Schematic representation of the OPG system

Two additional systems combine with the MOR to form the OPG. The 48 temporally-shaped and modulated pulses become the inputs to 48 preamplifier modules (PAMs). The PAM is a large, self-contained laser amplifier system that serves multiple functions. A PAM amplifies the $1 \mathrm{~nJ}$ input pulse from the MOR up to $22 \mathrm{~J}$ (for an unshaped pulse), which is the largest gain increment in the NIF laser. Amplification is accomplished with a diodelaser pumped, Nd-doped glass, regenerative amplifier(regen), and a large, 4-pass rod amplifier. The PAMs spatially reshape the Gaussian beam that is injected from the MOR and amplified by the $\mathrm{TEM}_{00}$ regen to match the aperture shape of the main amplifiers and precompensate for their spatial gain profile. The if bandwidth that is added in the 
MOR for SSD is spatially dispersed in the PAMs. In addition to these primary functions, the PAMs perform several auxiliary tasks: the 4-pass, in conjunction with the PABTS, isolates the rest of the PAM from backward traveling pulses from the main amplifiers; the regen subsystem contains an energy limiter to prevent too large a pulse from being injected into the main laser chain, and the regen Pockels cell slicer cleans up the temporal pulse shape reducing prepulses to an acceptable level.

The final system in the OPG is the Preamplifier Beam Transport System (PABTS). In the PABTS, the 48 PAM outputs are each split four ways to form 192 (4X48) pulses that are the inputs for the 192 main amplifier chains. The 4-way division of the $15 \mathrm{~J}$ spatially-shaped, PAM output injects up to $3 \mathrm{~J}$ into each amplifier chain at the transport spatial filter injection point. Beam size and temporal delay can be adjusted in each PAB'TS beamline with a zoom telescope and optical delay line. A large aperture $(55 \mathrm{~mm}$ ) Faraday isolator is used to help protect the PAMs from a high energy, backward propagating pulse from the main amplifier chain.

Much of the development of individual subsystems within the MOR and PAM systems is complete and in many cases engineering prototyping is underway. In the past year we have devoted part of our effort toward integrated performance demonstrations where we have linked the completed systems in the MOR and Preamplifier Laboratories into an $O P G$ Testbed. We use the OPG Testbed to demonstrate the combined performance of the two active systems within the OPG and to verify, experimentally, comprehensive models that describe the energetics and propagation of a pulse through the OPG.

\section{DESCRIPTION}

The requirements for the OPG system flowdown from the overall laser system requirements at the target. Table I lists the overall system requirements at the target and the resulting parameters for the OPG system determined from the flowdown.

NIF System Requirements [1]

$\begin{array}{ll}\text { Output energy } & 1.8 \mathrm{MJ} \\ \text { Peak power } & 500 \mathrm{TW} \\ \text { Wavelength } & 352 \mathrm{~nm} \\ \text { Pulse duration } & 20 \mathrm{~ns} \\ \text { Power balance (in 2 ns window) } & 8 \% \\ \text { Power dynamic range } & >50: 1 \\ \text { Prepulse in } 20 \text { ns window } & <10^{\wedge} 8 \mathrm{~W} / \\ \text { Number of beamlets } & 192 \\ & \\ \text { Optical Pulse Generation Requirements } & \\ \text { Injected energy into main amps } & 3.0 \mathrm{~J} \\ \quad \text {-Preamp output energy (flattop beam) } & 22 \mathrm{~J} \\ \quad \text {-Preamp power energy (shaped beam) } & 14.7 \mathrm{~J} \\ \text { Peak power at injection } & 1.2 \mathrm{GW} \\ \text { Wavelength } & 1053 \mathrm{~nm} \\ \text { Pulse duration } & 20 \mathrm{~ns} \\ \text { Power balance (in 2 ns window) } & <3 \% \\ \text { Power dynamic range } & >125: 1 \\ \quad-\text { Square-pulse-distortion } & <2.3 \\ \text { Prepulse contrast } & >2 \mathrm{X} 10^{\wedge} 6 \\ \text { Number of preamplifier modules } & 48\end{array}$

Table I. System requirements for the laser at the larget and the requirements at the output of the OPG determined from a flowdown of the system requirements back to the Front End.

The OPG Testbed is a skeleton version of the combined MOR and PAM systems that we assembled to demonstrate the performance requirements listed in Table I. In the NIF MOR the original pulse is generated by the fiber master oscillator [2], a Q-switched, Yb-doped fiber, ring oscillator that produces a $130 \mathrm{~mW}$ pulse at a $960 \mathrm{~Hz}$ repetition rate. We chop a $30 \mathrm{~ns}$ square pulse from the $300 \mathrm{~ns}$ pulsed output of the oscillator to reduce the laser 
energy and saturation in the fiber amplifiers. The single frequency oscillator output is phase modulated in two stages to add FM bandwidth for SBS suppression and SSD [3]. A $3 \mathrm{GHz}$ integrated optic modulator adds $30 \mathrm{GHz}$ of bandwidth for SBS suppression. A second modulator is driven at $17 \mathrm{GHz}$ to add $80-150 \mathrm{GHz}$ of FM bandwidth for SSD. The next component in the MOR is the amplifier tree where the single line is divided into 48 parallel lines via a series of 4-way splitters and fiber amplifiers. The fiber amplifiers are two-stage amplifiers that also use Yb-doped fiber. For the OPG Testbed we hooked up three of these amplifiers in series, replicating a single one of the 48 parallel lines. The final components in the MOR are the 48 pulse shapers [3] that carve the desired temporal pulse shapes from the $30 \mathrm{~ns}$ square pulse chopped from the oscillator output. The shapes are produced electronically with high bandwidth arbitrary waveform generators (AWG). The electronic pulse shapes drive integrated optic modulators that carve the 48 optical pulses into the desired shapes. The outputs of the MOR feed pulses to the 48 preamplifiers via single-mode polarizing fiber.

The MOR layout for the OPG Testbed is slightly different than a NIF beamline as shown by the block diagram in Fig. 2. Since we have a single beamline we can easily interchange subsystems to test for problems. For example we know that FM-to-AM conversion is a problem in the MOR due to the large number of polarization sensitive fiber components [4], consequently we reduced the AM by placing the phase modulator at the end of the MOR chain. For the Testbed experiments we used a single, $3 \mathrm{GHz}$ modulator and added up to $81 \mathrm{GHz}$ of FM bandwidth. Although the AWG was available for the Testbed, we used a variable-width, square-pulse generator to drive the amplitude modulator (pulse chopper in Fig. 2) for the experiments discussed in Section 3.
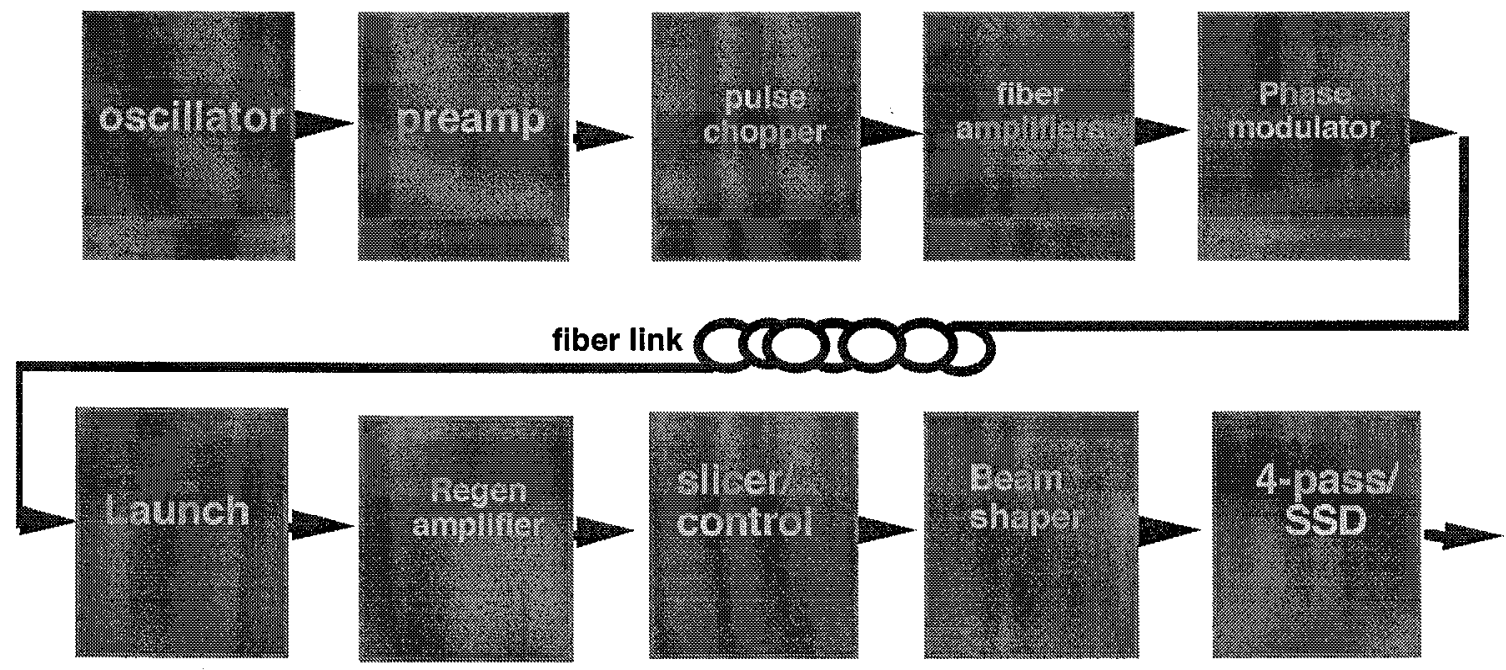

Figure 2. Block diagram of the Integrated OPG Testbed

The components of the preamplifier that are included in the Integrated OPG Testbed are also shown in Fig. 2. A diode-laser-pumped regenerative amplifier (regen) boosts the energy delivered to the preamplifier from the MOR from $1 \mathrm{~nJ}$ up to $20 \mathrm{~mJ}$, an amplification of $73 \mathrm{~dB}$ [5]. The regen has a single, diode-pumped amplifier that is pumped by a $4 \mathrm{~kW}$ diode array, producing a single pass gain of $1.5-1.65$. The regen operates at a $1 \mathrm{~Hz}$ repetition rate. The regen cavity is a long, folded $\mathrm{TEM}_{00}$ cavity that can cleanly switch in a pulse up to $24 \mathrm{~ns}$ long. The pulsc exiting the regen is sent through a Pockels cell slicer that trims off unwanted prepulses from the regen. The Gaussian spatial shape of the laser pulse leaving the regen is altered in the beam shaping module to form a square beam whose shape is tailored to compensate for the spatial gain profiles of the 4-pass and main amplifiers. This reshaping of the regen Gaussian beam is accomplished with a $20 \mathrm{X}$ telescope and 2 chrome-on-glass, variable transmission, spatial shaping filters. A third filter, the serrated aperture, apodizes the beam so it will propagate through the rest of the laser system with minimal edge ringing from diffraction. The serrated aperture is the reference plane that is image relayed through the 4-pass amplifier and the rest of the laser chain. The spatial shaping of the regen output reduces the PAM laser energy from $20 \mathrm{~mJ}$ down to $600 \mu \mathrm{J}$, which is the energy injected into the 4-pass amplifier. The 4-pass amplifier uses a Nova-style, flashlamp-pumped, $5 \mathrm{~cm}$ rod amplifier that can produce a singlepass gain of 25 using a specially clad rod. The 4-pass amplifier employs passive, polarization switching with a Faraday rotator, waveplate, and thin-film polarizers to achieve 4 passes through the amplifier. The pulse is relayed through the 4-pass system with $1: 1$ vacuum relay telescopes that are $2 \mathrm{~m}$. in length to eliminate temporal pulse 
overlap in the rod amplifier. The output of the 4-pass amplifier is $22 \mathrm{~J}$ for a flattop beam and $15 \mathrm{~J}$ for a spatially shaped beam. The final subsystem in the Preamplifier is the SSD optical system for spatially dispersing the frequency modulated light produced in the MOR. In earlier designs the SSD optics were a separate subsystem with a diffraction grating, relay telescope, waveplates and thin-film-polarizer. We have eliminated this separate SSD subsystem by incorporating a single diffraction grating into the 4-pass optical layout as shown in Fig. 3 . The grating at Littrow angle replaces the second cavity mirror in the 4-pass.

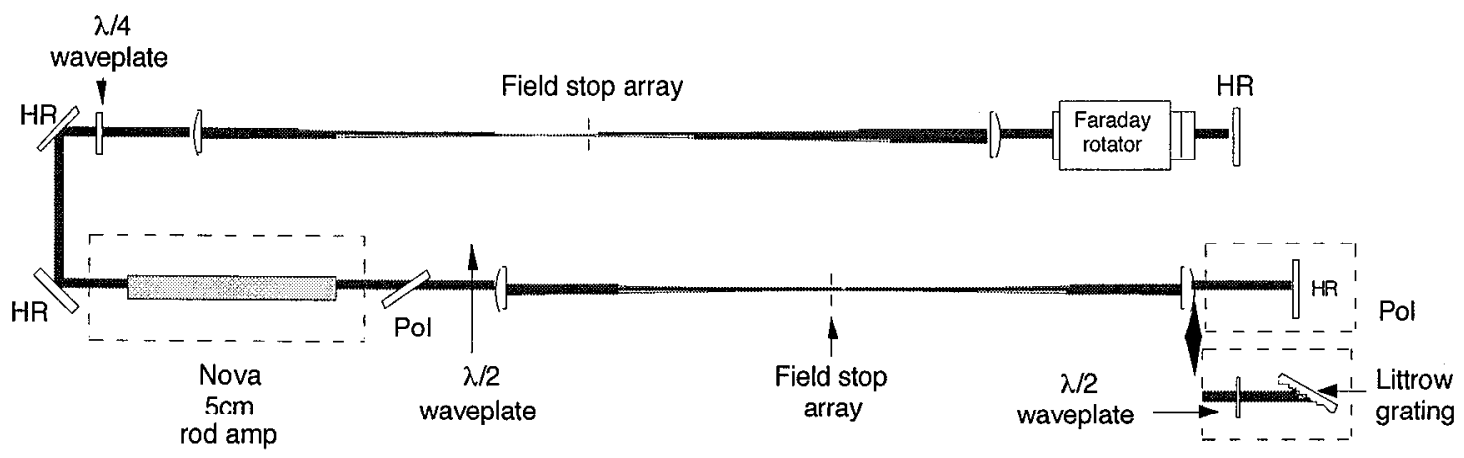

Figure 3. Layout of 4-pass amplifier with grating at Littrow for SSD

We set up several diagnostics throughout the OPG Testbed. We measure energy and power at the regen input, regen output, and 4-pass output with fast photodiodes and calorimeters. In addition we measure energy at the 4-pass input. We use cameras at the regen and 4-pass outputs to record the near-field and far-field images of the beam. We measure spectra in the MOR with a Fabry-Perot etalon, and at the output of the regen with a Fizeau spectrometer. All of the diagnostic outputs are acquired and stored on computers for further analysis.

\section{RESULTS}

We assembled the Integrated OPG Testbed to demonstrate the performance capabilities of the Front End as a combined system. Some requirements such as SSD depend on functioning subsystems in the MOR and Preamp labs. Many of the OPG specifications such as output energy, power, and temporal pulse contrast must be flowed back from the OPG output though the other major subsystems, 4-pass, regen, fiber amps, in order to determine the contributions of the component subsystems. Eventually, 48 PAMs will be built, and so it is important to determine how they perform with the MOR as a system, what the failure mechanisms are, what are the sources and magnitudes of variations in output energy and power. In conjunction with system mesurements we developed models that memic the energy, power, and propagation behavior of the OPG and employed these models to analyze system performance.

\subsection{Subsystem function}

We operated the OPG Testbed with all of the Preamplifier subsystems in place: regen, beam shaping, SSD with $81 \mathrm{GHz}$ FM bandwidth, and four-pass amplifier. The goal of this experiment was to demonstrate all of the functions of the preamp, and to prove that we could achieve the required output energy and square-pulse-distortion with high-contrast beam shaping and grating dispersion for SSD in place. The configuration for this experiment is represented by the block diagram in Fig. 2, and Fig. 3 shows the location of the SSD grating in the 4-pass optical layout.

The current beam smoothing requirement for the NIF is $3 \AA$ bandwidth, critically dispersed in onedimension. "Critically dispersed" means that the temporal skew produced across the $3 \mathrm{~cm}$ beam by the grating at Littrow angle must be less than or equal to the period of the sinusoidal r.f. waveform that drives the phase modulator. The temporal skew, $\Delta \mathrm{T}$, is given by

$$
\Delta T=2 D \tan \theta / c \leq 1 / f_{\bmod }
$$


where $\mathrm{D}=30 \mathrm{~mm}$ is the beam dimension at the grating in the dispersion direction, and $\mathrm{f}_{\bmod }$ is the r.f. driver frequency. The Littrow angle is given by $\theta=\sin ^{-1}(\lambda / 2 \mathrm{~d})$, where $\lambda$ is the wavelength of the laser and $\mathrm{d}$ is the grating groove spacing. In the current design the modulation frequency for SSD is $17 \mathrm{GHz}$, and the critical dispersion requirement is met for a 600 line/mm grating. The $17 \mathrm{GHz}$ modulator system was not available for the OPG subsystem tests so we used a $3 \mathrm{GHz}$ modulation frequency and an 1800 line/mm grating to meet the critical dispersion requirement.

We fired a number of shots at full output energy ( 15J) while increasing the FM bandwidth in steps by increasing the r.f. power to the phase modulator. Figure 4 shows the output of the four-pass amplifier in the farfield. The angular dispersion produced by the 1800 line/mm grating cleanly separates the individual spots in the farfield image. We show a plot in Fig. 4 comparing a lineout from the camera image with a power spectrum for an $81 \mathrm{GHz}$ modulation bandwidth. The power spectrum is given by the Bessel series:

$$
\begin{aligned}
& \mathbf{E}(\mathbf{f}) \approx \sum J_{n}(m) \cdot \delta\left(f_{0}+n f_{r f}\right) \\
& P(f) \approx|E|^{2},
\end{aligned}
$$

where, $\mathrm{E}(\mathrm{f})$ is the laser field in the frequency domain, $\mathrm{J}_{\mathrm{n}}$ is the $\mathrm{n}$-th order Bessel function, $\mathrm{m}$ is the modulation index, $\delta$ is the delta function, and $f_{\mathrm{rf}}$ is the rf frequency driving the phase modulator. The locations of the peaks and their magnitudes from the image lineout accurately compare with the simulated spectral peaks. The $81 \mathrm{GHz} F M$ spectra is confirmed by the spectrometer measurements in the MOR and at the regen output.
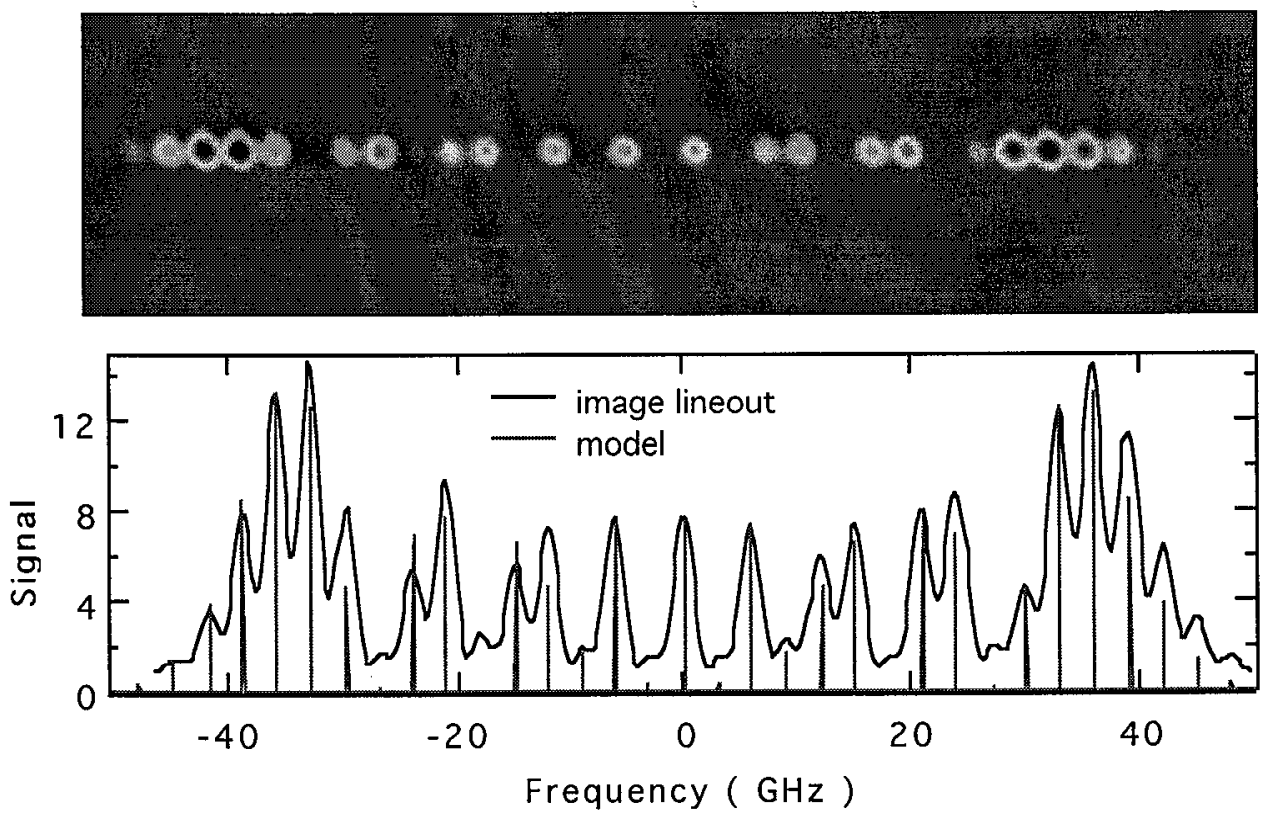

Figure 4. Far-field image at 4-pass output shows individual spots dispersed by grating in the 4-pass. The plot shows a comparison between a lineout from the far-field image and the power spectrum for an FM signal with $81 \mathrm{Ghz}$ of bandwidth and a $3 \mathrm{Ghz}$ modulation frequency.

We determine the desired spatial shape of the bcam that is injected into the 4-pass amplifier using a detailed propagation model for an entire NIF amplifier chain [ref]. The goal of the model and shaping design is to produce a flattop beam at the target. The model contains detailed two-dimensional files describing the large amplifier gain profiles, rod amplifier gain profile, lens files, vacuum spatial filters, and any other component that affects laser propagation. The model can produce accurate and detailed beam shapes anywhere along the propagation path, thus the model is useful for determing B-integral and fluence levels in key optical componenets in the chain. The ideal design for the beam shaper must take the Gaussian mode from the regen, magnify it in size to fill the necessary square aperture, then sculpt the beam to precompensate for the spatial gain profiles of the $5 \mathrm{~cm}$ rod amplifier in the 4-pass and the large, slab amplifiers in the main laser cavity, delivering the desired flattop fluence profile at the target. We 
employ three chrome-on-glass filters to shape the beam in the beam shaping module. We truncate the magnified Gaussian beam from the regen with an antiGaussian filter that flattens the top of the Gaussian shape. The second filter sculpts out the center of the beam to compensate for the gain profiles of the down stream amplifiers based on the propagation model design. A third filter, the serrated apodizer, places a specified roll-off on the edge of the beam to control edge ringing on the beam as it propagates through the rest of the laser system and produces the optimum fill of the square laser aperture. Figure 5 shows the measured nearfield image at the output of the 4-pass taken during an Integrated OPG Testbed experiment and the shape predicted by the propagation model [7]. The measured output profile and the model prediction are nearly identical.

\section{measured near-field output}

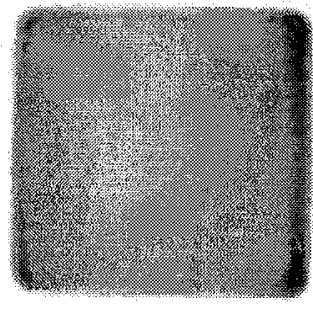

pseudo-color bearn Image

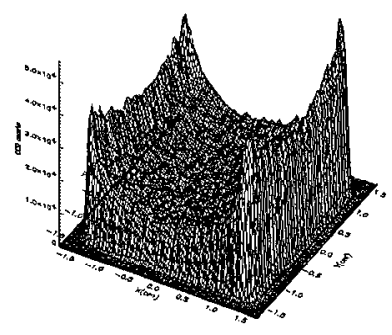

surface plot
Prop model simulation
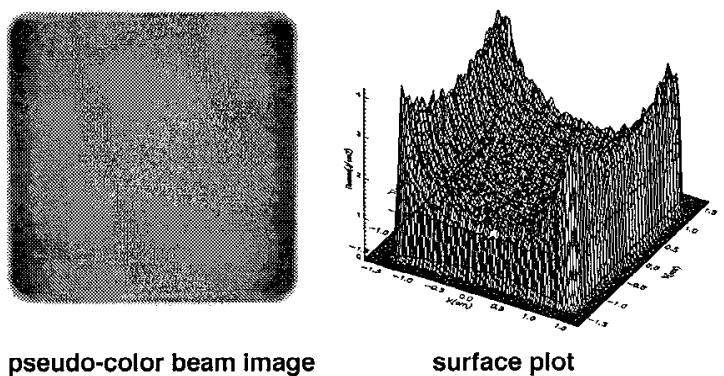

Figure 5. Comparison between measured and modeled near-field images for a $16 \mathrm{~J}$ shot

\subsection{Energy and power flowdown}

The energy, power, and temporal pulse distortion specifications for the Front End flowdown from the target requirements shown in Table I. These specifications are for an indirect drive, ICF mission, and may vary for different target experiments and missions (e.g. direct drive). The output energy and power specifications for the PAM are based upon a requirement of $3.0 \mathrm{~J}$ in a high-contrast, spatially-shaped beam at the transport spatial filter of a main amplifier chain. The 3.0 J OPG output corrcsponds to a $14.7 \mathrm{~J}$ PAM output based upon the equal, four-way power split and the optics specifications for the PABTS. We have a separate specification of $22 \mathrm{~J}$ at the PAM output for a flattop beam.

We generated a detailed energetics and power model for the entire Front End that includes the losses of all the optical components from the master oscillator in the MOR to the injection mirror for the transport spatial filter in the main amplifier chain. We enter the optical component losses for a single beamline into a spread sheet that includes the gain and saturation characteristics of each amplifier in a chain. The time-dependent gain, $G(t)$, for an amplifier is calculated using [Siegman],

$$
\mathbf{G}(\mathbf{r}, \mathbf{t})=\underset{1-\left(1-(1 / \mathbf{G}(\mathbf{r}, 0)) \cdot \exp \left(-\mathbf{U}(\mathbf{r}, \mathbf{t}) / \mathbf{J}_{\mathbf{s}} \mathbf{t}\right)\right.}{1}
$$

where $\mathrm{U}(\mathrm{r}, \mathrm{t})$ is

$$
U(r, t)=\int_{0}^{t} \mathbf{I}\left(r, t^{\prime}\right) d t^{\prime}
$$

and

$$
I_{\text {out }}(r, t)=G(r, t) \cdot I(r, t)
$$

and $I(r, t)$ is the irradiance of the pulse at the amplifier input, $G(r, 0)$ is the initial, radially-dependent gain, and Jsat is the saturation fluence of the amplifier transition. We calculate output power and energy by integrating the output irradiance over time and space. We determine the square-pulse-distortion (SPD) from the time-dependent gain, $G(t)$. We corroborate the accuracy of each amplifier model by comparison with amplifier characterization measurements. In Figure 6 we plot measured values of output energy from the 4-pass amplifier vs input energy for a fixed gain of 
16.0. For this measurement we injected a spatial beam shape produced by filling a $30 \mathrm{~mm}$ square aperture with a magnified Gaussian beam from the beam shaper telescope. This input shape produces an approximately flattop beam at the 4-pass output. The solid line in the plot shows the model predictions for the same parameters measured in the experiment. These results show that we can easily meet the $22 \mathrm{~J}$ requirement for a square flattop beam.

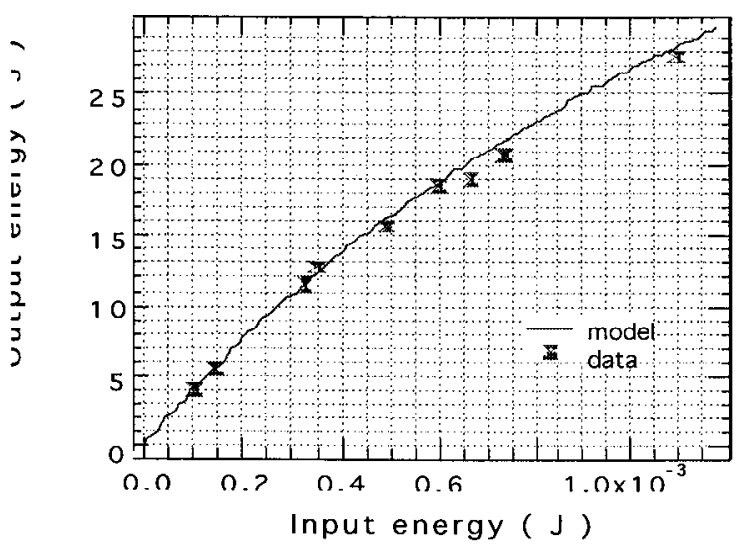

Figure 6. Graph shows 4-pass output energy vs input energy, data and model.

We measured the energy, power, and temporal pulse distortion at three locations on the Integrated OPG Testbed to demonstrate the performance specifications given in Table I and to compare our model predictions to measured values. We used fast photodiodes $(T<100 \mathrm{psec})$ at the PAM input, regen output, and 4-pass output. We acquired the photodiode signals on a Tektronix TDS 684 oscilloscope that has a $1 \mathrm{Ghz}$ bandwidth per scope channel. We calibrated each photodiode signal to an energy calorimeter and converted the photodiode voltage to laser power. In addition we measured the energy at the 4-pass input. For the measurements we operated the MOR with $81 \mathrm{GHz}$ of FM bandwidth and included both the beam shaping and the $600 \mathrm{l} / \mathrm{mm}$ grating placed in the 4-pass layout at $\mathrm{I}$ ittrow angle. With all of the PAM subsystems in place we can compare our measured results with the flowdown model for energy through the OPG and evaluate our design.

a.

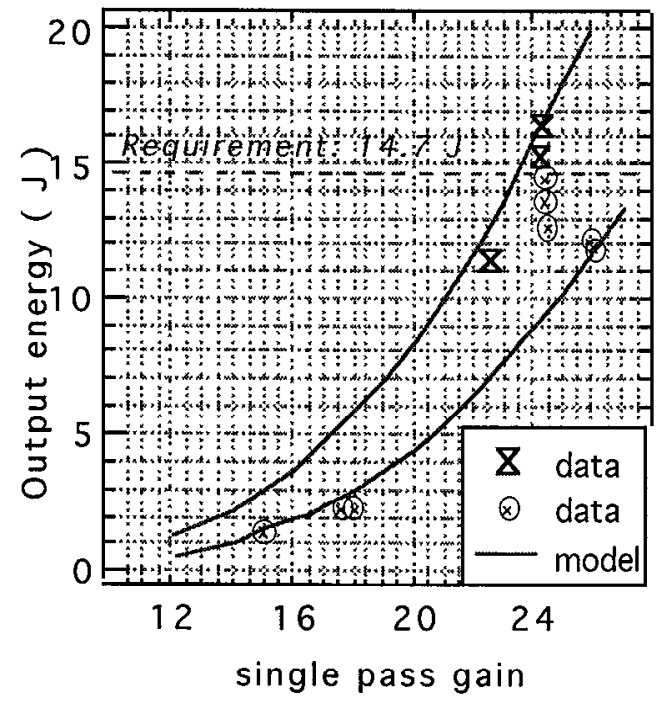

b.

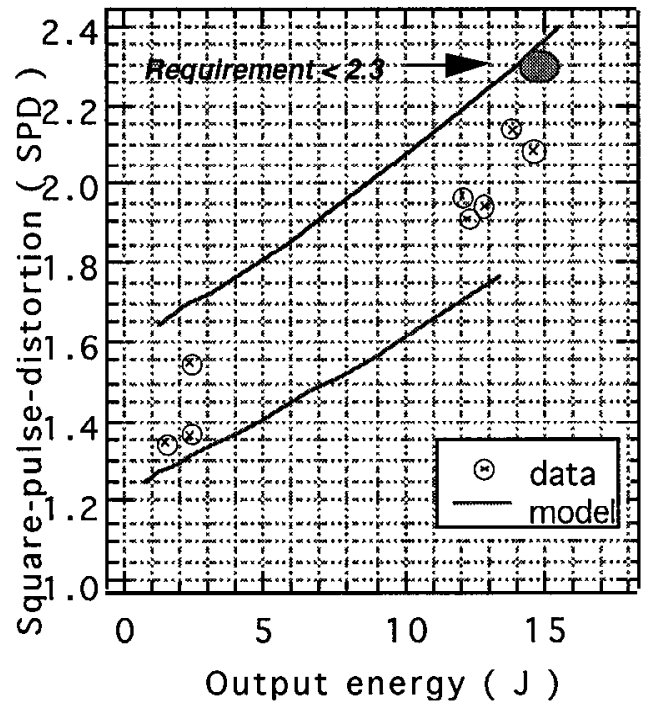

Figure 7. a. Graph plots output energy vs $5 \mathrm{~cm}$ rod amplifier gain; b. graph plots SPD vs output energy

Figure 7 shows plots of the PAM output energy vs rod amplifier gain and square-pulse-distortion vs output energy from the 4-pass. In these measurements we held the regen gain constant and fired the system for different values of $5 \mathrm{~cm}$. rod amplifier gain. The PAM output energy varies for constant gain because the injected energy from the MOR is varying. The red curves on each plot in Fig. 7 show the output predicted by the detailed energetics 
model: the lower curve uses the lowest value of MOR energy injected into the PAM for this experiment and the upper curve uses the largest injected MOR energy. For both model curves we used the measured gain and transmission values for the PAM. The model curves bracket most of the data points in either plot and verify the accuracy of the model in predicting results on the Integrated OPG Testbed.

Quantity

1. MOR injection energy

2. Launch transmission

3. Regen gain

4. Regen output energy

5. Slicer transmission

6. Beam shaper transmission

7. 4-pass transmission $\mathrm{w} /$ mirror

8. 4-pass transmission w/ grating

$9.5 \mathrm{~cm}$ rod gain

10. PAM square-pulse-distortion

11. PAM output energy
Measured value

$60-180 \mathrm{pJ}$

0.61

$80-85 \mathrm{~dB}$

$12-23 \mathrm{~mJ}$

0.60

0.02

0.55

0.23

24

2.1

13-16.5 J
Flowdown value

$710 \mathrm{pJ}$

0.61

$74 \mathrm{~dB}$

$20 \mathrm{~mJ}$

0.40

0.077

0.55

0.47

19

$<2.3$

$14.7 \mathrm{~J}$

Table II. Summary of energetics flowdown experiment

Table II shows a summary of the results from the energetics flowdown experiment plus the design flowdown value for the measured quantity. Some values such as launch and 4-pass transmission are identical because the design value is based upon the measured value. The measured slicer transmission is higher than the design value because we plan to add a second Pockels cell slicer for additional prepulse extinction and to limit high output pulses from the regen. Additional losses in the beamshaper telescope, beam shaper module and the poor efficiency of the $6001 / \mathrm{mm}$ SSD grating lowered the system transmission after the regen. We were still able to meet the output energy goal by increasing the rod amplifier gain from 19 to 24 . We believe that we can reduce these PAM tranmission losses with improved mask designs for the the beam shaper and a higher efficiency grating in the 4-pass amplifier.

\subsection{Power balance}

To accomplish the goals of the target physicists the NIF laser system is designed to illuminate either the inside of a cylindrical hohlraum, or a spherical target with a high degree of uniformity. This illumination uniformity requirement is translated into a power balance specification for the entire laser system and a portion of that power balance budget is given to the OPG system as listed in Table I. The power imbalance is specified by Eq. $(6,7)$,

$$
\begin{aligned}
& \{\mathrm{dP}(\mathrm{t})\}_{\mathrm{i}}=\int_{2 \mathrm{~ns}}\left[\left(\mathrm{P}_{\mathrm{i}}(\mathrm{u})-\mathrm{P}_{\text {ref }}(\mathrm{u})\right) / \mathrm{P}_{\mathrm{ref}}(\mathrm{u})\right] \mathrm{du} \\
& \{\mathrm{dP}(\mathrm{t})\}_{\mathrm{rms}}=\sqrt{ }\left\{\Sigma[\mathrm{dP}(\mathrm{t})]_{\mathrm{i}}{ }^{2} / 192\right\}
\end{aligned}
$$

$\mathrm{Eq}(6)$ defines the power imbalance between the measured output of a single beam and a reference pulse shape, $\mathbf{P}_{\text {ref, }}$, that is specified for the particular target experiment. The power imbalance is calculated for any 2 ns window over the duration of the pulse.[O.J.] The power imbalance for 192 beams is averaged as shown in Eq(7) to give the final result. For the OPG system, $\{\mathbf{d P}(\mathbf{t})\}_{\mathrm{rms}}<\mathbf{0 . 0 3}$.

Several different quantities throughout the OPG system contribute to the power imbalance between the output pulse and the reference pulse. Energy fluctuations in the oscillator and laser amplifiers in the MOR and PAM, errors in pulse shaping in the arbitrary waveform generator (AWG) or amplitude modulator, timing jitter in the AWG trigger, imbalance among the legs in the PABTS after the 4-way split, imbalance or transmission fluctuations among separate lines in the 1-to-48 split in the MOR, and errors in the propagation model defining the digital shape that is input to the AWG can all contribute to an imbalance between a single pulse shape and the reference pulse shape, or among the 48 different beam lines. By measuring the variations in the contributing subsytems within the OPG system we can determine an error budget that divides the 3\% total OPG system contribution among the MOR, PAM and PABTS. Since the Integrated OPG Testbed is a single beamline we cannot test differences between two or 
more outputs, however we operated the Testbed at a fixed operating point and measured changes about that set point, and determined the variations of the components subsystems.

a.

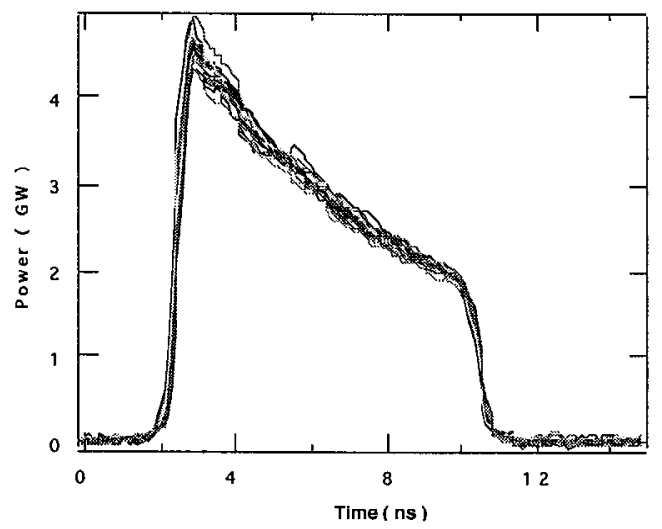

b.

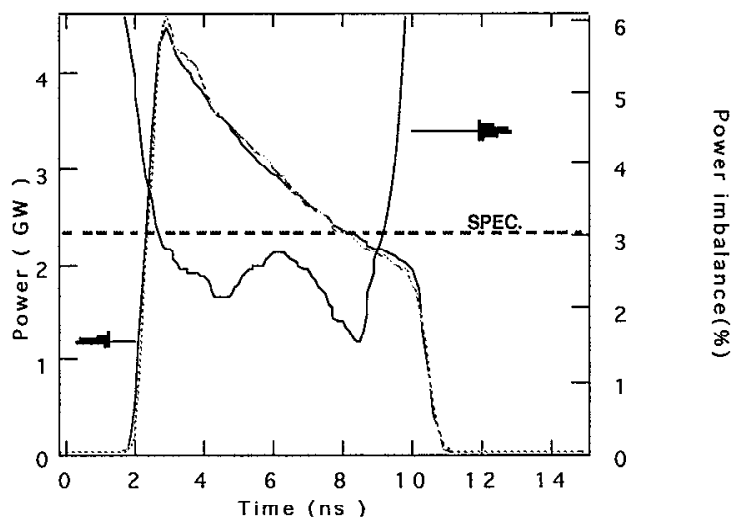

Figure 8a. Plot shows 18 successive PAM output pulses overlayed. b. Comparison between the average of the 18 pulses in $8 \mathrm{a}$ and the energetics model output; also a plot of the rms power balance between the 18 pulses in $8 \mathrm{a}$ and the average pulse in $8 b$.

Figure 8a shows 18 successive pulses measured at the output of the Testbed overlayed to demonstrate the repeatability of the system when operated in steady-state. The average energy of these 18 pulses was $24 \mathrm{~J}$, exceeding the performance specification listed in Table I for an unshaped pulse. Figure $8 \mathrm{~b}$ shows two sets of pulses: the set that goes with the right-hand ordinate shows the average of the 18 pulses in 8a plotted with the energetics model output using the measured parameters for gain, loss, and injected energy for the steady state operation; the curve that goes with the left-hand ordinate shows the power imbalance calculated for the 18 shots using Eqs. 6,7, with the average of the 18 pulses as the reference pulse in the formula (solid curve). In Table III we present a summary of the measured values for the 18 successive shots shown in Fig. 8

1. average PAM input energy

2. input energy standard deviation

3. average regen output energy

4. regen energy standard deviation

5. regen gain per pass

6. average regen $S P D$

7. average PAM output energy

8. PAM output standard deviation

9. average rod amplifier gain

10 gain standard deviation

11. average PAM SPD

12 average electrical energy to rod amp

13. electrical energy standard deviation

14. flashlamp explosion fraction

15. pulse jitter measured at PAM output

16. number of shots

17. PAM repetition rate

Table III. Summary of PAM steady state operation
$680 \mathrm{pJ}$

$33.5 \mathrm{pJ}(4.9 \%)$

$11.74 \mathrm{~mJ}$

$0.50 \mathrm{~mJ}(4.3 \%)$

1.60

1.24

$24.1 \mathrm{~J}$

$0.54 \mathrm{~J}(2.2 \%)$

16.75

$0.08(0.48 \%)$

2.20

$20.39 \mathrm{~kJ}(0.22 \%)$

$45 \mathrm{~J}$

0.20

$150 \mathrm{psec}$

18

shot/10 min.

Fluctuations in the MOR energy injected into the regen and the output variation of the regen are $4.9 \%$ and $4.3 \%$ respectively. However the overall variation of the PAM output energy is only $2.2 \%$ because amplifier saturation reduces the fluctuations seen at the 4-pass input. The rms power imbalance shown in Fig. $8 \mathrm{~b}$ also falls below the $3 \%$ specification for all but the leading and trailing edges of the pulse. We will perform a more rigorous power 
balance test on the Integrated $\mathrm{OPG}$ l'estbed using a high-contrast, shaped, temporal pulse that has a long (12 ns) "foot" that is $1 / 275$ of the peak power. For such a high-contrast pulse, the foot remains in the linear amplification regime through the entire PAM amplifier, so variations in gain in the regen and MOR will not be reduced by saturation in the 4-pass amplifier. Also, a highly-shaped input pulse tests other sources of error such as timing jitter and the ability to predict the electronic input pulse that produces the desired OPG system output laser pulse. We are currently investigating methods to reduce gain and energy fluctuations in the MOR and regen [6].

\section{SUMMARY}

We assembled an Integrated OPG Testbed by combining the MOR and Preamplifier Development Labs. We used this facility to test the overall performance of the NIF OPG system up to the PABTS input. We measured and demonstrated most of the performance specifications for the OPG system, especially energy, power, and pulse contrast capabilities. We continue to work on improving the Testbed performance in arcas such as beam shaping, and power balance, and we are developing an output diagnostic instrument to accurately measure wavefront at the 4-pass output. We developed comprehensive models for energetics and beam propagation through the OPG system and demonstrated the capability of these models to replicate experimental results. Based upon these measurements we have a solid design for the PAM and MOR systems and are near completion of the first PAM engineering prototype[7].

\section{ACKNOWLEDGMENTS}

Work done under the auspices of the U. S. Department of Energy by Lawrence Livermore National Laboratory under contract No. W-7405-Eng-48. The authors received valued advice from several colleagues including Ken Skulina, Jim Davin, Raymond Beach, Ken Manes, Scott Mitchell, Jay Skidmore, Curt Laumann, and John Braucht.

\section{REFERENCES}

1. J. A. Paisner, J. D. Boyes, S. A. Kumpan, W. H. Lowdermilk, M. Sorem, "Conceptual Design of the National Ignition Facility", Proc. 1st International Conf. On Solid State Lasers for Application to Inertial Confinement Fusion, Monterey, 2633, p. 2 SPIE Proceedings Series Bellingham, WA, 1995.

2. S. C. Burkhart, R. J. Beach, J. K. Crane, J. M. Davin, M. D. Perry, R. B. Wilcox, "The National Ignition Facility Front-End Laser System", Proc. 1st International Conf. On Solid State Lasers for Application to Inertial Confinement Fusion, 2633, p. 48 SPIE Proceedings Series Bellingham, WA, 1995.

3. S. C. Burkhart, R. Wilcox, D. Browning, F. Penko, "Amplitude and Phase Modulation with Waveguide Optics", Proc. Ist International Conf. On Solid State Lasers for Application to Inertial Confinement Fusion, Monterey, 3047, p. 610-617, SPIE Proceedings Series Bellingham, WA, 1998.

4. J. E. Rothenberg...."FM-to-AM...", Proc. 3rdt International Conf. On Solid State Lasers for Application to Inertial Confinement Fusion, Monterey, to be published, SPIE Proceedings Series Bellingham, WA.

5. M. D. Martinez, J. K. Crane, L. A. Hackel, F. Penko, D. Browning, "Optimized, diode-pumped, Nd:glass, Prototype Regenerative Amplifier for the National Ignition Facility", Proc. on Optoelectronics and HighPower Lasers \& Applications, San Jose, CA 24 -30 Jan 1998, SPIE Proceedings Series.

6. B. D. Moran, C. B. Dane, J. K. Crane, M. D. Martinez, F. Penko, L. A. Hackel, "Suppression of Parasitics and Pencil Beams in the High-Gain National Ignition Facility Multipass Preamplifier", Proc. on Optoelectronics and High-Power Lasers \& Applications, San Jose, CA 24 -30 Jan 1998, SPIE Proceedings Series. 
7. M. Henesian, et. al., "Propagation modeling of the NIF Laser System", Proc. 3rd International Conf. On Solid State Lasers for Application to Inertial Confinement Fusion, ????, p. ?? SPIE Proceedings Series Bellingham, WA, 1998.

8. O. S. Jones, D. R. Speck, S. W. Haan, "The NIF Power Balance", Proc. 3rd International Conf. On Solid State Lasers for Application to Inertial Confinement Fusion, ????, p. ?? SPIE Proceedings Series Bellingham, WA, 1998.

9. N. W. Hopps, R. B. Wilcox, M. R. Hermann, M. D. Martinez, E. H. Padilla, J. K. Crane, "Optimissation of the alignment sensitivity and energy stability of the NIF regenerative ampifier", Proc. 3rd International Conf. On Solid State Lasers for Application to Inertial Confinement Fusion, ????, p. ?? SPIE Proceedings Series Bellingham, WA, 1998.

10. M. D. Martinez, K. M. Skulina,... "NIF Preamplifier Prototype", Proc. 3rd International Conf. On Solid State Lasers for Application to Inertial Confinement Fusion, ????, p. ?? SPIE Proceedings Series Bellingham, WA, 1998. 